\title{
Evaluation of total body water in canine breeds by single-frequency bioelectrical impedance analysis method: specific equations are needed for accuracy
}

Laurence Yaguiyan-Colliard ${ }^{1 *}$, Caroline Daumas ${ }^{2}$, Patrick Nguyen², Dominique Grandjean ${ }^{1}$, Philippe Cardot ${ }^{3}$, Nathalie Priymenko ${ }^{4}$ and Françoise Roux ${ }^{5}$

\begin{abstract}
Background: Equations based on single-frequency bioelectrical impedance analysis at $50 \mathrm{kHz}$ for determination of total body water content (TBW) have been previously validated in healthy non-sedated beagle dogs. We investigated whether these equations are predictive of TBW in various canine breeds by comparing the results of these equations with TBW values evaluated directly by deuterium oxide $\left(\mathrm{D}_{2} \mathrm{O}\right)$ dilution.

Methods: Total body water content of 13 healthy adult pet dogs of various breeds was determined directly using $\mathrm{D}_{2} \mathrm{O}$ dilution and indirectly using previous equations based on values obtained with a portable bioelectric impedance device. Paired Student's t-tests were used to compare TBW obtained by single-frequency bioelectrical impedance analysis and $\mathrm{D}_{2} \mathrm{O}$ dilution. A $\mathrm{p}$-value of $<0.05$ was considered statistically significant for all analyses.

Results: Significant differences were observed between TBW determined by the reference method and the values obtained with both predictive equations.

Conclusions: The proposed equations including single-frequency bioelectrical impedance analysis parameters validated at $50 \mathrm{kHz}$ in healthy adult beagles need to be modified including morphological parameters such as body size and shape in a first approach. As in humans, morphological-specific equations have to be developed and validated.
\end{abstract}

Keywords: Dogs, Bioimpedance analysis, Deuterium dilution, Total body water, Body composition

\section{Background}

Determination of total body water content (TBW) and body composition analysis are essential for meaningful medical follow-up of individuals [1] in physiological (e.g., growth, aging, and sport) as well as pathological (e.g., obesity, dialysis, and disability) states. Therefore, simple and effective techniques for evaluation of body composition are highly desirable.

Bioelectrical impedance analysis (BIA) is often presented as an alternative to dilution techniques and to

\footnotetext{
*Correspondence: nutrition@colliard.fr

1 Breeding and Sport Medicine Unit, Université Paris-Est, École Nationale Vétérinaire d'Alfort, 7 avenue du Général de Gaulle, Maisons-Alfort 94704, France

Full list of author information is available at the end of the article
}

dual X-ray absorptiometry for the evaluation of TBW and body composition. It is a quick non-invasive technique that requires inexpensive equipment and a low-intensity electrical current, which is painless and undetectable by the subject. BIA has been validated and routinely used in healthy as well as sick people [2-4]. This technique has also been validated with various types of equipment in several species including dogs [5-8], which has made it possible to derive linear regression equations for TBW evaluation. Nevertheless, development of specific equations in humans based on sex, age, ethnic group, and physical activity has been necessary [9]. No similar data for dogs have been published.

Because BIA has potential as an easy technique for assessment of body composition in veterinary practice, 
we aimed to verify whether the protocol and equations validated in adult non-anesthetized beagle dogs for single-frequency (SF-BIA) at $50 \mathrm{kHz}$ [8] were applicable for the prediction of TBW in non-anesthetized dogs of various breeds.

\section{Methods}

Subjects

Thirteen adult dogs of various breeds (3 middle-sized crossbred dogs, 2 Australian shepherd dogs, 2 Siberian huskies, 2 golden retrievers, 1 miniature schnauzer, 1 Yorkshire terrier, 1 Belgian shepherd dog, and 1 English bulldog) owned and voluntarily provided by veterinary students were used in the study. A written informed consent was obtained from each owner. The dogs were $4.3 \pm 2.9$ years (mean $\pm \mathrm{SD}$ ) in age and weighed $20.97 \pm 8.09 \mathrm{~kg}$ (mean $\pm \mathrm{SD})$. The mean body condition score (9-point scale BCS) was 5.3/9 with a range of 5/9 to $7 / 9$. The experimental protocols adhered to European Union ethical guidelines and were approved by the Institute of Animal Clinical Research of Alfort.

\section{Deuterium dilution technique}

Food and water were restricted from $2 \mathrm{~h}$ before to $4 \mathrm{~h}$ after injection of the tracer. TBW was measured using the isotopic dilution of deuterium $\left(\mathrm{D}_{2} \mathrm{O}\right)$. On the day of the measurement, 12-h food-deprived dogs were injected subcutaneously in the dorsal cervical region with $\mathrm{D}_{2} \mathrm{O}$ [99.9\% $\mathrm{H} / \mathrm{H}^{2} ; 500 \mathrm{mg} / \mathrm{kg}$ body weight (W); Eurisotop, Gif-sur-Yvette, France] prepared with physiological saline solution $(9 \mathrm{~g} \mathrm{NaCl} / \mathrm{L})$. Syringes were weighed before and after administration of $\mathrm{D}_{2} \mathrm{O}$ to determine the exact dose administered, with an accuracy of $0.1 \mathrm{~g}$. Blood samples $(5 \mathrm{~mL})$ were collected in EDTA tubes from the jugular vein before and $4 \mathrm{~h}$ after injection of the tracer [10]. Plasma was separated by centrifugation at $2,000 \times g$ for $10 \mathrm{~min}$, and stored at $-20^{\circ} \mathrm{C}$ in sealed vials until analysis. Plasma $\mathrm{D}_{2} \mathrm{O}$ was assayed in duplicate using Fourier-transform infrared spectroscopy, as validated and described previously by Ferrier et al. [11].

\section{Blood testing}

Blood samples $(2 \mathrm{~mL})$ were collected in heparin tubes from the jugular vein before injection of the tracer for determination of hematocrit and blood glucose, total protein, albumin, sodium, potassium, and chloride levels.

\section{Body measurements}

Following injection of the tracer, the animals were weighed and measured. BCS for each dog was evaluated by the same trained veterinarian. Body length ( $\mathrm{L}$; from the external occipital protuberance to the base of the tail) was determined using a flexible tape. Thorax (rib-cage) circumference
(RC; at the xyphoid process) and abdominal circumference (AC; at the umbilicus) were measured using a self-rewind tape (Holtex+, Aix-en-Provence, France) when the animal was in full expiration. Scapula height $(\mathrm{H})$ was measured from the ground to the dorsal border of the scapula with a portable stadiometer (Tanita France SA, Neuilly-sur-Seine, France). All measurements were recorded in centimeters $( \pm 1 / 10)$ and weights in kilograms $( \pm 1 / 100)$.

\section{SF-BIA}

The animal was placed in a non-electrically conductive harness and then connected to the equipment through four electrodes clamped on the right fore- and hindlimb as previously described by Yaguiyan-Colliard et al. [8]. The dog did not have to be restrained by an operator. The structure was placed on an electrically insulating mat so the animal did not contact any electrically conductive object.

Each animal was subjected to three sequential measurements of impedance with the bioelectrical impedance device (Z-Métrix, BioparHom, Bourget du Lac, France) with a $77 \mu \mathrm{A}$ current and $50 \mathrm{kHz}$ frequency. The dogs were restrained in the harness for about $5 \mathrm{~min}$ for the procedure. Sequential measurements also enabled investigation of the repeatability of the measurements. Data were transmitted directly from the analyzer to a computer and stored in a spreadsheet format file (Microsoft Office Excel, Microsoft France, Issy-les-Moulineaux, France).

\section{Statistical analysis}

Repeatability of measurements refers to the variation in repeat measurements made on the same subject under identical conditions. Variability in measurements made on the same subject can then be ascribed only to errors related to the measurement process itself. The repeatability of $\mathrm{R}$ and $\mathrm{X}$ measurements in each of the 13 dogs in the study was evaluated by the coefficient of variation $(\mathrm{CV})$ as 100 times the ratio between the standard deviation (SD) and the mean of the $\mathrm{R}$ and $\mathrm{X}$ measurements, respectively.

The two predictive equations for TBW (1) and (2) were previously obtained by linear regression and validated in adult beagle dogs [8].

$$
\begin{aligned}
\mathrm{TBW}_{1}= & -0.019(\mathrm{~L} / \mathrm{R})+-0.199(\mathrm{RC}+\mathrm{AC}) \\
& +0.996 \mathrm{~W}+0.081 \mathrm{H}+12.31 \\
\mathrm{TBW}_{2}= & 0.048(\mathrm{~L} / \mathrm{R})+-0.144(\mathrm{RC}+\mathrm{AC}) \\
& +0.777 \mathrm{~W}+0.066 \mathrm{H}+0.031 \mathrm{X}+7.47]
\end{aligned}
$$

The TBW values computed using impedance measurements $\left(\mathrm{TBW}_{\mathrm{i}}\right)$ with the two equations and the TBW value measured directly by isotopic dilution $\left(\mathrm{TBW}_{\mathrm{d}}\right)$ 
were calculated. We used paired Student's $t$-tests to compare TBW obtained by SF-BIA and dilution (reference method). A p-value of $<0.05$ was considered statistically significant for all analyses.

\section{Results}

The owners' dogs remained quiet in the restraining device without operator interference and tolerated the electrodes for the duration of the measurements.

Blood test results were found to be within normal ranges in all the dogs (data not shown).

The physical characteristics of the dogs and values obtained (Table 1) were used to calculate the TBW by using the previously validated Eqs. (1) and (2).

The median CV was $2.90 \%$ (range $0.27-9.54 \%$ ) for $\mathrm{R}$ and $2.38 \%$ (range $0.05-10.56 \%$ ) for X (Table 2).

Applying the two equations to the group of dogs demonstrated an overestimation of TBW compared with data obtained by isotopic dilution. The mean difference was $1.96 \pm 2.04 \mathrm{~L}$ ( Diff $_{1}$ ) for the first equation and $3.31 \pm 5.06 \mathrm{~L}\left(\mathrm{Diff}_{2}\right)$ for the second equation (Table 2 ). This corresponds to differences of 14.4 and $22.2 \%$ between computed $\mathrm{TBW}_{1}$ and $\mathrm{TBW}_{2}$, respectively, and $\mathrm{TBW}_{\mathrm{d}}$.

Student's $t$-tests were applied to the values obtained by the two equations. The values obtained by dilution were significantly different from those obtained using SF-BIA $\left(\mathrm{p}_{1}=0.0046\right.$ for the first equation and $\mathrm{p}_{2}=0.036$ for the second equation) (Table 2).

\section{Discussion}

A simple system was developed in the previous study for restraint for SF-BIA measurements in beagles [8]. This system efficiently maintained the study dogs of various breeds standing on four legs in a physiological position, without any discomfort, for the few seconds the measurements required. This is an important point because the envisaged future benefit of this study is the use of BIA in field conditions.

In this study, SF-BIA at $50 \mathrm{kHz}$ was applied in nonlaboratory healthy adult dogs of various breeds. The BIA system could be applied in non-sedated dogs without generating unnecessary stress in the animals. The TBW values obtained with predictive equations based on BIA and morphological parameters were significantly different from those obtained with $\mathrm{D}_{2} \mathrm{O}$ dilution.

In our previous study on 26 beagle dogs with the same protocol and device [8], the mean CVs were slightly lower than those of the present study (1.6 vs. $2.9 \%$ for $\mathrm{R}$ and 2.2 vs. $2.38 \%$ for X). However coefficients of variation for $\mathrm{R}$ and $\mathrm{X}$ found in this study were low, which illustrates that the precision of the BIA method was good with the study dogs in spite of the variety of breeds and coats, and a lower compliancy than laboratory dogs.

Bioelectrical impedance analysis may be affected by many variables such as hydration status, consumption of food and water, skin and air temperature, recent physical activity, conductance of the examination table, and posture, in addition to electrode positioning and instrumentation [12-14]. This study was designed to standardize and control as many of these variables as possible. No alterations were found in blood parameters in any of the dogs that could have altered the conduction of electric current in the body (data not shown).

Table 1 Parameters of the 13 dogs

\begin{tabular}{|c|c|c|c|c|c|c|c|c|}
\hline Dogs & Breed & Sex & Age (years) & $W(k g)$ & $\mathrm{L}(\mathrm{cm})$ & $\mathrm{RC}(\mathrm{cm})$ & $A C(\mathrm{~cm})$ & $\mathrm{H}(\mathrm{cm})$ \\
\hline 1 & Mixed breed & M & 7.00 & 32.50 & 90 & 76.6 & 55 & 64.4 \\
\hline 2 & Australian shepherd & $M N$ & 3.75 & 18.95 & 69.2 & 63.3 & 50.3 & 56.6 \\
\hline 3 & Australian shepherd & M & 3.00 & 28.30 & 73.8 & 70.8 & 55.8 & 52.3 \\
\hline 4 & Siberian husky & FN & 1.00 & 17.10 & 70.3 & 62.6 & 46.3 & 52.5 \\
\hline 5 & Miniature schnauzer & M & 1.30 & 6.95 & 40.2 & 45.5 & 36.4 & 36.2 \\
\hline 6 & Mixed breed & $\mathrm{MN}$ & 2.00 & 21.50 & 64.8 & 66.2 & 53.5 & 50.7 \\
\hline 7 & Golden retriever & M & 6.50 & 27.80 & 76.1 & 73.3 & 56.6 & 54.7 \\
\hline 8 & Yorkshire terrier & M & 10.90 & 5.15 & 43.7 & 40.2 & 34.1 & 26.1 \\
\hline 9 & Golden retriever & MN & 4.70 & 27.30 & 79.6 & 71.8 & 58 & 58.8 \\
\hline 10 & Siberian husky & M & 2.70 & 19.50 & 80.3 & 62.2 & 45.9 & 58.6 \\
\hline 11 & Mixed breed & FN & 4.10 & 17.65 & 61.7 & 62 & 49.3 & 48.7 \\
\hline 12 & Belgian shepherd dog & FN & 2.20 & 25.60 & 75.6 & 70.5 & 51.9 & 60.3 \\
\hline \multirow[t]{3}{*}{13} & English bulldog & FN & 7.20 & 24.25 & 58 & 65.9 & 56.4 & 35.9 \\
\hline & & Mean & 4.33 & 20.97 & & & & \\
\hline & & SD & 2.86 & 8.09 & & & & \\
\hline
\end{tabular}


Table 2 Bioimpedance parameters and TBW values obtained in the study dogs with the two methods

\begin{tabular}{|c|c|c|c|c|c|c|c|c|c|c|c|}
\hline Dogs & $\mathrm{R}(\Omega)$ & $\mathrm{SD}_{\mathrm{R}}(\Omega)$ & $\mathrm{CV}_{\mathrm{R}}$ & $X(\Omega)$ & $\mathrm{SD}_{\mathrm{X}}(\Omega)$ & $\mathrm{CV}_{\mathrm{x}}$ & $\mathrm{TBW}_{\mathrm{d}}(\mathrm{L})$ & $\mathrm{TBW}_{1}$ (L) & Diff $_{1}(\mathrm{~L})$ & $\mathrm{TBW}_{2}$ (L) & Diff $_{2}(\mathrm{~L})$ \\
\hline 1 & 97.87 & 0.30 & 0.30 & 22.70 & 0.24 & 1.08 & 19.46 & 22.14 & 2.68 & 22.65 & 3.19 \\
\hline 2 & 188.42 & 1.48 & 0.77 & 37.65 & 0.28 & 0.73 & 11.41 & 12.68 & 1.27 & 11.91 & 0.50 \\
\hline 3 & 127.78 & 4.00 & 3.14 & 32.95 & 3.48 & 10.56 & 12.60 & 18.73 & 6.13 & 17.70 & 5.10 \\
\hline 4 & 164.83 & 12.46 & 7.56 & 59.52 & 0.03 & 0.05 & 6.35 & 11.35 & 5.00 & 11.77 & 5.42 \\
\hline 5 & 108.70 & 0.29 & 0.27 & 27.75 & 0.22 & 0.79 & 4.45 & 5.58 & 1.13 & 4.99 & 0.53 \\
\hline 6 & 84.68 & 0.99 & 1.17 & 21.12 & 0.17 & 0.80 & 13.11 & 13.07 & -0.04 & 13.27 & 0.16 \\
\hline 7 & 23.43 & 2.24 & 9.54 & 298.51 & 5.52 & 1.85 & 15.90 & 13.88 & -2.02 & 35.04 & 19.14 \\
\hline 8 & 135.00 & 2.75 & 2.04 & 27.86 & 1.13 & 4.05 & 3.01 & 4.50 & 1.49 & 3.99 & 0.98 \\
\hline 9 & 100.13 & 1.64 & 1.64 & 36.04 & 0.56 & 1.56 & 15.78 & 17.23 & 1.45 & 17.98 & 2.20 \\
\hline 10 & 148.38 & 1.21 & 0.81 & 33.49 & 0.29 & 0.86 & 12.10 & 14.14 & 2.04 & 14.00 & 1.89 \\
\hline 11 & 142.84 & 4.44 & 3.11 & 33.06 & 0.53 & 1.60 & 8.51 & 11.18 & 2.67 & 10.63 & 2.12 \\
\hline 12 & 117.06 & 5.83 & 4.98 & 27.30 & 0.72 & 2.64 & 15.01 & 17.41 & 2.39 & 16.86 & 1.84 \\
\hline 13 & 104.77 & 2.44 & 2.33 & 17.77 & 0.78 & 7.38 & 13.15 & 14.42 & 1.27 & 13.11 & -0.04 \\
\hline Mean $\pm S D$ & & & 2.90 & & & 2.38 & & & $1.96 \pm 2.04$ & & $3.31 \pm 5.06$ \\
\hline
\end{tabular}

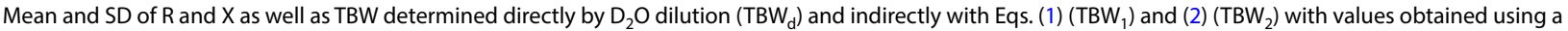
portable bioelectric impedance device [resistance $(\mathrm{R})$ and reactance $(\mathrm{X})$ ] and morphological parameters. Diff ${ }_{1}$ and Diff ${ }_{2}$ : differences between the TBW $\mathrm{d}_{\mathrm{d}}$ value and the TBW values computed with Eqs. (1) and (2), respectively.

However, the diversity of shapes and coats of canine breeds as well as the body condition of the dogs might explain the inaccuracy of predictive equations for TBW validated in beagle dogs observed in this study. One dog, a Braque Français cross (dog no. 6; Tables 1, 2), showed similar results for TBW between the two methods. This dog, although larger, had the same shape and coat as a beagle dog.

\section{Conclusions}

Body shape and condition as overweight impacted the results, albeit the dogs used in this study had an average normal body condition.

This study showed that in dogs, as in humans, it is necessary to define predictive equations for TBW based on BIA parameters according to various morphological parameters such as body size and body condition. Further study is needed to determine coefficients for the equations in dogs according to variables such as breed, sex, condition, activity level, and age.

\section{Abbreviations}

AC: abdominal circumference; BCS: body condition score; BIA: bioimpedance analysis; $\mathrm{CV}$ : coefficient of variation; $\mathrm{D}_{2} \mathrm{O}$ : deuterium; $\mathrm{H}$ : scapula height; $\mathrm{L}$ : body length; R: resistance; RC: rib-cage circumference; SD: standard deviation; SFBIA: single-frequency bioelectrical impedance analysis; TBW: total body water; W: body weight; $X$ : reactance; Z: impedance.

\section{Authors' contributions}

LYC and FR instigated the study and participated in the study design; acquisition, analysis, and interpretation of data; and drafting of the manuscript. CD participated in the study design and acquisition, analysis, and interpretation of data. PN participated in analysis and interpretation of data. DG participated in drafting of the manuscript. PC and NP participated in the study design and drafting of the manuscript. All authors read and approved the final manuscript.

\section{Author details}

${ }^{1}$ Breeding and Sport Medicine Unit, Université Paris-Est, École Nationale Vétérinaire d'Alfort, 7 avenue du Général de Gaulle, Maisons-Alfort 94704, France.

${ }^{2}$ Nutrition and Endocrinology Unit, École Nationale Vétérinaire de Nantes (ONIRIS), Atlanpole La Chantrerie, route de Gachet, CS 40706, 44307 Nantes Cedex 3 , France. ${ }^{3}$ Experimental and Clinical Respiratory Neurophysiology Unit, Faculté de Médecine Pierre and Marie Curie, Université Pierre et Marie Curie, UMRS-1158, 91 Boulevard de l'Hôpital, 75634 Paris Cedex 13, France. ${ }^{4}$ École Nationale Vétérinaire UMR1331 Toxalim INRA/INP/UPS-ENVT, BP 87614, 31076 Toulouse Cedex 3, France. ${ }^{5}$ Intensive Care Medicine Unit, École Nationale Vétérinaire de Nantes (ONIRIS), Atlanpole La Chantrerie, route de Gachet, CS 40706, 44307 Nantes Cedex 3, France.

\section{Acknowledgements}

We would like to express our gratitude to IDEXX France for free supply of biochemical analysis plates.

This manuscript represents a part of the requirement for a $\mathrm{PhD}$ degree that will be presented by Dr. Yaguiyan-Colliard to the doctoral school ED 394 Physiology, Physiopathology, and Therapeutics Department, Pierre and Marie Curie University.

\section{Compliance with ethical guidelines}

\section{Competing interests}

The authors declare that they have no competing interests.

Received: 7 January 2015 Accepted: 22 July 2015

Published online: 06 August 2015

\section{References}

1. Thibault R, Pichard C (2012) The evaluation of body composition: a useful tool for clinical practice. Ann Nutr Metab 60:6-16

2. Jaffrin MY (2009) Body composition determination by bioimpedance: an update. Curr Opin Clin Nutr Metab Care 12(5):482-486

3. Casey AF (2013) Measuring body composition in individuals with intellectual disability. J Obes. doi:10.1155/2013/628428 
4. Böhm A, Heitmann BL (2013) The use of bioelectrical impedance analysis for body composition in epidemiological studies. Eur J Clin Nutr 67:S79-S85

5. Sheltinga MR, Helton WS, Rounds J, Jacobs DO, Wilmore DW (1991) Impedance electrodes positioned on proximal portions of limbs quantify fluid compartments in dogs. J Appl Physiol 5:2039-2044

6. Burkholder WJ (1994) Body composition of dogs determined by carcass composition analysis, deuterium oxide dilution, subjective and objective morphometry, and bioelectrical impedance. Ph.D. thesis, Virginia Polytechnic Institute and State University, Blacksburg, p 357

7. Free Patents Online Web Site (2014) Pet body fat measuring tool: United States patent application 20090076408. http://www.freepatentsonline. com/20090076408.pdf. Accessed 2 Dec 2014

8. Yaguiyan-Colliard L, Daumas C, Bousbiat S, Jaffrin M, Cardot P, Grandjean $D$ et al (2015) Indirect assessment of total body water in adult beagle dogs by single-frequency bioelectrical impedance analysis (SF-BIA). Am J Vet Res 76:547-553
9. Ward LC (2012) Segmental bioelectrical impedance analysis: an update. Curr Opin Clin Nutr Metab Care 15:424-429

10. Jeusette I, Daminet S, Nguyen P, Shibata H, Saito M, Honjoh T et al (2006) Effect of ovariectomy and ad libitum feeding on body composition, thyroid status, ghrelin and leptin plasma concentrations in female dogs. J Anim Physiol Anim Nutr 90:12-18

11. Ferrier L, Robert $P$, Dumon H, Martin L, Nguyen P (2002) Evaluation of body composition in dogs by isotopic dilution using a low-cost technique, Fourier-transform infrared spectroscopy. J Nutr 132:1725S-1727S

12. Gudivaka R, Schoeller DA, Kushner RF, Bolt MJG (1999) Single- and multifrequency models for bioelectrical impedance analysis of body water compartments. J Appl Physiol 87(3):1087-1096

13. O'Brien C, Baker-Fulco CJ, Young AJ, Sawka MN (1999) Bioimpedance assessment of hypohydration. Med Sci Sports Exerc 31:1466-1471

14. Scharfetter H, Monif M, László Z, Lambauer T, Hutten H, Hinghofer-Szalkay $H$ (1997) Effect of postural changes on the reliability of volume estimations from bioimpedance spectroscopy data. Kidney Int 51:1078-1087

\section{Submit your next manuscript to BioMed Central and take full advantage of:}

- Convenient online submission

- Thorough peer review

- No space constraints or color figure charges

- Immediate publication on acceptance

- Inclusion in PubMed, CAS, Scopus and Google Scholar

- Research which is freely available for redistribution

Submit your manuscript at

www.biomedcentral.com/submit

C Biomed Central 